\title{
Preparation and In Vitro Characterization of Artemisinin Freeze-Dried Powders with Various Carriers
}

Gamal Osman Elhassan ${ }^{1,2^{*}}$, Yuen Kah Hay ${ }^{3}$, Wong Jia Woei ${ }^{3}$, Jiyauddin Khan ${ }^{4}$, Khalid Omer Alfarouq $^{5}$, Javed Akhter ${ }^{1}$, Habibullah Khalilullah $^{1}$, MU Khan ${ }^{1}$, Riyaz Ahmed Khan ${ }^{1}$ and Kamal Ahmad Qureshi ${ }^{1}$

${ }^{1}$ Unaizah College of Pharmacy, Qassim University, Qassim, Kingdom of Saudi Arabia

${ }^{2}$ Faculty of Pharmacy, Omdruman Islamic University, Khartoum, Sudan

${ }^{3}$ School of Pharmaceutical Sciences, Universiti Sains Malaysia, Penang, Malaysia

${ }^{4}$ Management and Science University, Selangoor, Malaysia

5 Institute of Endemic Diseases, University of Khartoum, Khartoum, Sudan

"Corresponding author: Gamal Osman Elhassan, Unaizah College of Pharmacy, Qassim University, Qassim, Kingdom of Saudi Arabia, Tel: 00966530965955; E-mail: gamaosma63@yahoo.com

Rec date: Nov 24, 2014; Acc date: Dec 22, 2014; Pub date: Dec 29, 2014

Copyright: ( 2014 Elhassan GO, et al. This is an open-access article distributed under the terms of the Creative Commons Attribution License, which permits unrestricted use, distribution, and reproduction in any medium, provided the original author and source are credited.

\begin{abstract}
The present study was conducted to investigate the enhancing effect of different polymeric carriers on solubility and hence oral bioavailability of artemisinin, a poorly water-soluble drug. Freeze-dried powder preparations of artemisinin and various carriers (polyvinyl pyrrolidone K-25 (PVP K-25), Hydroxypropyl Cellulose (HPC) and dextrin) were obtained by dissolving different carriers (PVP K-25, HPC and dextrin) in water, followed by the addition of artemisinin at a ratio of 1:4. The resultant products were evaluated using solubility and dissolution studies, Differential Scanning Calorimetry (DSC) and Scanning Electron microscopy (SEM). These in vitro studies showed that the aqueous solubility of artemisinin was significantly increased for the preparation containing artemisinindextrin at a ratio of 1:4. Further, the effect of incorporation of different co-carriers (citric acid or mannitol) to artemisinin-dextrin freeze-dried powder at different ratios was evaluated. A significant increase in the solubility and dissolution rate of artemisinin was obtained with the artemisinin-dextrin-citric acid freeze-dried powder at a ratio of 1:3:1.
\end{abstract}

Keywords: Artemisinin; HPC; PVP K-25; Dextrin; Solubility

\section{Introduction}

Artemisinin (ART) is the active principle isolated from the Chinese medicinal herb Artemisia annua [1]. ART and its derivatives have gained increasing attention as antimalarial drugs due to their low toxicity and high efficacy against malarial parasites, including the cases caused by multidrug resistant and cerebral strains. It is one of the few antimalarials that remain effective against multi drug-resistant strains of Plasmodium falciparum [2]. It is a fast acting blood schizonticide with short parasite clearance time, leading to rapid symptomatic relief of malarial infections [3].

The major drawback of this compound is the low aqueous solubility, resulting in poor and erratic absorption upon oral administration. This together with its short half-life and high first pass metabolism might lead to incomplete clearance of parasites resulting in recrudescence [4].

To overcome the problem of poor solubility and dissolution of artemisinin, various approaches have been reported. One of these approaches is the freeze-drying (lyophilization) technique, which is widely used in the pharmaceutical industry. Tachibana and Nakuma [5] were among the first to dissolve both the drug and the carrier in common solvent and then evaporate the solvent to produce a solid mixture. Jaccard and Leyder [6] employed freeze-drying technique in making oral pharmaceutical preparations and found that the products have increased absorption and bioavailability with poorly water soluble drugs like spironolactone, nicergoline and trolendymein in comparison to their respective conventional formulations. Betageri and Makarla reported that lyophilized solid dispersions of glybenclamide-PEGs had the maximum effect on the rate and extent of dissolution of glybenclamide compared to their physical mixtures. The results clearly suggested that inclusion of a carrier together with suitable lyophilization process can have a positive influence on poorly water-soluble drugs.

Van Eerdenbrugh et al. [7] reported that the dissolution rate and absorption properties of loviride administered as sucrose co-freezedried nano-powder were improved significantly. The use of freezedrying techniques to enhance the solubility, dissolution rate and bioavailability of poorly water-soluble drugs (nimesulide, nifedipine, griseofulvin and loviride) has been demonstrated by Shoikri et al. [8], Iman \& Mona [9] and Van Eerdebrugh et al. [7], respectively.

The aim of the present study was to enhance the aqueous solubility and the dissolution rate of artemisinin using freeze-drying techniques.

\section{Materials}

Artemisinin was obtained from Kuming Pharmaceutical Corporation (Kuming, Germany). Dextrin, citric acid and mannitol were obtained from BDH Chemicals (Poole, England). All others chemicals and reagents used were either analytical or HPLC grades. 


\section{Methods}

\section{Preparation of artemisinin freeze-dried powders with different carriers}

Freeze-dried powders containing different proportions of artemisinin to Polyvinyl Pyrrolidone (PVP K-25), Hydroxylpropy Cellulose (HPC) and dextrin, were prepared using ratios of drug to carrier of 1:4, 2:3, 3:2 and 4:1. They were prepared by first dissolving each carrier in distilled water followed by the addition of artemisinin with constant heating at $70-80^{\circ} \mathrm{C}$ and stirring. Dextrin is not soluble in most common solvents, but it is highly soluble in hot water.

Another set of experiments were done by adding co-carriers citric acid or mannitol into the artemisinin-dextrin mixture. They were prepared by dissolving Dextrin with citric acid / mannitol in hot distilled water, followed by addition of artemisinin with constant heating at $60^{\circ} \mathrm{C}-80^{\circ} \mathrm{C}$. Various ratios of ART: dextrin: citric acid / mannitol were prepared; the composition details are shown in Table 1.

\begin{tabular}{|l|l|l|l|}
\hline Ratio & $\begin{array}{l}\text { Artemisinin } \\
(\mathbf{m g})\end{array}$ & $\begin{array}{l}\text { Dextrin } \\
(\mathbf{m g})\end{array}$ & $\begin{array}{l}\text { Citric Acid or } \\
\text { Mannitol }(\mathbf{m g})\end{array}$ \\
\hline $1: 3: 1$ & 100 & 300 & 100 \\
\hline $1: 2.75: 1.25$ & 100 & 275 & 125 \\
\hline $1: 3.25: 0.75$ & 100 & 325 & 75 \\
\hline
\end{tabular}

Table 1: Composition details of artemisinin: dextrin: citric acid/ mannitol used for the preparation of freeze dried powder.

All the above-mentioned mixtures were prepared and freezed at $-53^{\circ} \mathrm{C}$ for 24 hours before lyophilization. The frozen mixtures were transferred to the freeze dryer (Labconco freeze dryer, Labconco Corporation, USA) under medium vacuum $(8-10 \mathrm{~mm} \mathrm{Hg})$ and condensed at $-75^{\circ} \mathrm{C}$ for a period of 24 hours. The lyophilized powders were stored in a dessicator at room temperature prior to their characterization by SEM, DSC and solubility determination.

\section{Determination of artemisinin solubility in the prepared freeze-dried powders}

The solubility of artemisinin in the above prepared freeze-dried powders was determined. An excess amount of pure artemisinin and freeze-dried powder were separately added into flasks containing 20 $\mathrm{ml}$ of distilled water. All the samples were shaken vigorously at $30^{\circ} \mathrm{C}$ for 24 hours, $5 \mathrm{ml}$ samples were collected from each flask and filtered through a membrane filter $(0.2 \mu \mathrm{m})$. The filtrates were then suitably diluted and treated prior to analysis by High Performance Liquid Chromatography (HPLC) using an Ultraviolet (UV) detector operated at a wavelength of $260 \mathrm{~nm}$. The chromatographic separation was performed using a Genesis C18 column ( 150 x $4.6 \mathrm{~mm}$ ) (Genesis, UK). The mobile phase was composed of a mixture of $0.01 \mathrm{M}$ disodium hydrogen phosphate adjusted to $\mathrm{pH} 6.5$ and acetonitrile $(75: 25, \mathrm{v} / \mathrm{v})$. The HPLC analysis was based on the method reported by Zhao \& Zeng [10].

\section{Differential scanning calorimetry (DSC) studies}

Thermal analysis using DSC has proven to be a useful tool in characterizing the freeze-dried powders [11,12]. Differential scanning calorimetric studies were performed for all the freeze-dried powders with a TA instrument model 2010 differential scanning calorimeter (DE, USA). Each sample (10 $\mathrm{mg}$ of powder in aluminum pans) was scanned from 25 to $200^{\circ} \mathrm{C}$ at a rate of $10^{\circ} \mathrm{C}$ per minute, and the data were analyzed using the Universal analysis software (TA instrument, USA).

\section{Scanning electron microscopy (SEM) studies}

The images of artemisinin and all the above-mentioned freeze-dried powders were obtained by using a scanning electron microscope, and the micrographs were taken at a magnification of 500X.

\section{Dissolution study}

The in vitro dissolution studies of pure artemisinin, freeze-dried powders of artemisinin-dextrin-citric acid, artemisinin-dextrinmannitol, and their corresponding Physical Mixtures (PM) were evaluated using the paddle method with a USP 24 dissolution testapparatus (Sotax AT7, Basel, Switzerland).

The test was performed in $900 \mathrm{ml}$ of distilled water as the dissolution medium under non-sink condition. The temperature was maintained at maintained at $25^{\circ} \mathrm{C} \pm 0.5^{\circ} \mathrm{C}$ while the paddle rotation speed was set at $100 \mathrm{rpm}$. The temperature of $25^{\circ} \mathrm{C}$ was chosen because at a higher temperature artemisinin would recrystallize out when the samples are exposed to room temperature after collection. A weight of $250 \mathrm{mg}$ of pure artemisinin was used for each vessel. An equivalent weight of $250 \mathrm{mg}$ of artemisinin was also used for freeze-dried powders of artemisinin-dextrin-citric acid or mannitol and their corresponding physical mixture.

All powders were sieved through a $300 \mu \mathrm{m}$ laboratory test sieve (Endecotts Ltd., England) prior to the dissolution studies. Samples of 5 $\mathrm{ml}$ were withdrawn at various designated time intervals of $15,30,60$, $90,120,180,240,300$ and 360 minutes, using an automatic fraction collector (SDX Fractional Collector, Sadex, Malaysia). The samples were filtered through $0.2 \mu \mathrm{m}$ syringe membrane filters (Whatman, UK). The initial portion of the filtrate was discarded and the subsequent portion collected was subjected to appropriate dilution with distilled water prior to analysis by the HPLC method. For each sample, the dissolution test was run in triplicate.

\section{Results and Discussion}

\section{Preparation of artemisinin freeze-dried powders in different carriers}

The freeze-drying process could be completed in 24 hours. After 24 hours, the freeze-dried powder residues appeared as a porous light and fluffy mass, except for the preparation containing HPC, which was very sticky in nature, difficult to be collected and processed, and hence was excluded from further study.

\section{Determination of artemisinin solubility}

Figure 1 shows the solubility of artemisinin in the artemisinincarrier freeze-dried powders with different ratios of artemisinin to dextrin or PVP K-25. 


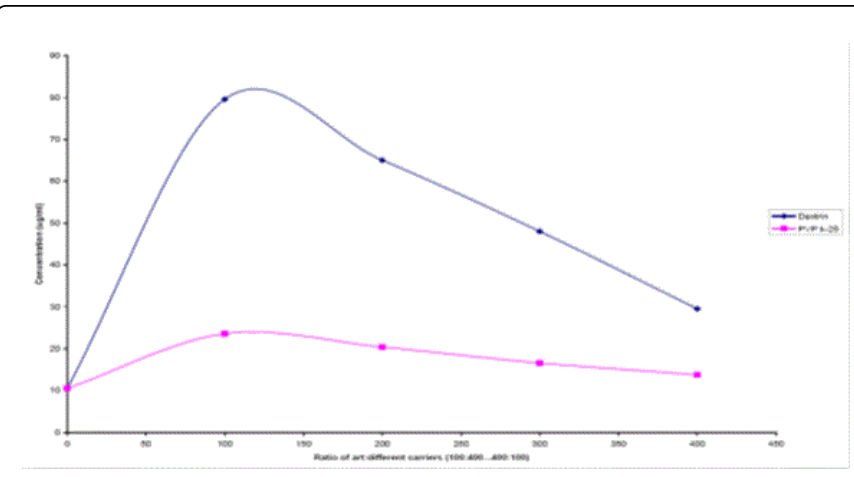

Figure 1: Comparative solubility profiles of artemisinin in different carriers (Dextrin/PVP K-25) prepared using water as a solvent.

It can be noticed that the solubility of artemisinin was influenced by the type and amount of carrier used. Dextrin was able to increase the solubility of artemisinin to a greater extent than PVP K-25 at all drug to carrier ratios used. The above-mentioned results showed that not only the type of the carriers used was important, but also the amount used can influence the drug solubility [13]. It is evident that at a ratio of $4: 1$ drug to carrier the solubility of artemisinin was optimum. The solubility of artemisinin in both carriers at a ratio of 4:1 is shown in Table 2 .

\begin{tabular}{|l|l|}
\hline Preparation & Solubility $(\mu \mathrm{g} / \mathrm{ml})$ \\
\cline { 2 - 3 } & Mean \pm SD, $\mathbf{n}=\mathbf{3}$ \\
\hline Pure artemisinin & $10.50 \pm 0.03$ \\
\hline Freeze-dried powder of artemisinin - Dextrin & $79.55 \pm 0.05$ \\
\hline $\begin{array}{l}\text { Freeze-dried powder of artemisinin - PVP } \\
\text { K-25 }\end{array}$ & $23.45 \pm 0.03$ \\
\hline
\end{tabular}

Table 2: Solubility of artemisinin in pure artemisinin and artemisinin carrier at a ratio of drug to carrier 1:4.

\section{Differential scanning calorimeter (DSC) studies}

Figure 2 and 3 represent the DSC thermograms of pure artemisinin, PVP K-25, dextrin, physical mixture of artemisinin-PVP K-25 / artemisinin-dextrin, and freeze-dried product of artemisinin-PVP K-25 / artemisinin-dextrin, respectively.

In Figure 2, it can be noticed that the characteristic peak of artemisinin was clearly visible in the physical mixture and freeze-dried product of artemisinin-PVP K-25 even in those powders with a high amount of PVP K-25 (1:4), indicating that there was no interaction between PVP K-25 and artemisinin. These results indicate that artemisinin was still in the crystalline form.

From the DSC thermograms shown in Figure 3, it can be noticed that there was an endothermic peak at $157.7^{\circ} \mathrm{C}$ for artemisinin, while for dextrin an endothermic peak was observed at $75^{\circ} \mathrm{C}-125^{\circ} \mathrm{C}$, indicating the presence of residual moisture in dextrin. In case of physical mixture of artemisinin-dextrin, the endothermic peak of artemisinin was intact with a negligible decrease in enthalpy of melting from 63.1 to $61.50 \mathrm{~J} / \mathrm{g}$, indicating that there was no interaction between artemisinin and dextrin.

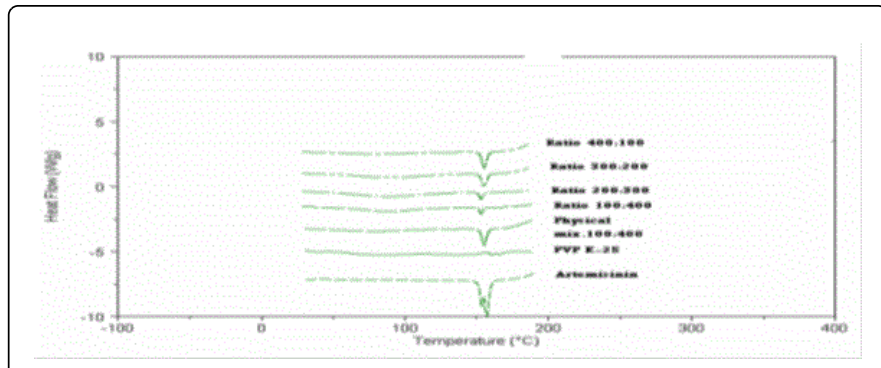

Figure 2: Thermograms of artemisinin and artemisinin-PVP K-25 at different ratio prepared using water as solvent.

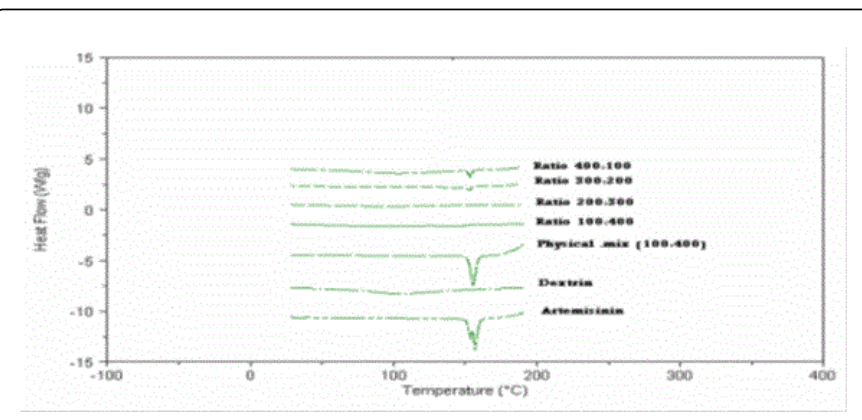

Figure 3: Thermograms of artemisinin and artemisinin-dextrin at different ratios.

In the case of freeze-dried product of artemisinin-dextrin, it was noticed that at a ratio of $1: 4$ and 2:3, the characteristic peak of artemisinin in the thermogram was absent. This indicates that artemisinin was no longer present in the crystalline form but was converted into the amorphous state [14]. The disappearance of the thermal features of the drug in the DSC thermogram indicated that the drug and the dextrin interacted with each other [15]. Similar findings have been reported by Te Wierk et al. [16] in the freeze-drying powder of diazepam with amylodextrin.

At ratios of 3:2 and 4:1 of artemisinin-dextrin, it can be noticed that a small peak of artemisinin was recorded in the DSC thermogram, indicating the presence of artemisinin still in the crystalline form.

\section{Scanning electron microscopy (SEM) studies}

Figure 4 illustrated the SEM micrographs of artemisinin, PVP K-25, dextrin, their physical mixture and freeze-dried product of artemisinin-PVP K-25 / dextrin. From the micrographs it can be noted that dextrin existed as small oval shaped particles, while in the physical mixture of artemisinin-dextrin the characteristic crystals of artemisinin were apparent. The freeze-dried product of artemisininPVP K-25 also showed the characteristic of artemisinin crystals, but these crystals were smaller in size. On the contrary, the freeze-dried product of artemisinin-dextrin appeared in the form of irregular particles in which the original morphology of both components was not visible at the ratio $1: 4$ and 2:3. But when the ratio of artemisinin to dextrin was increased to (3:2) and (4:1), the characteristic crystals of artemisinin were clearly visible, as shown in Figure 5. Thus, based on the results obtained from the above-mentioned DSC, SEM and solubility studies, the artemisinin-dextrin freeze-dried product at the ratio of 1:4 was selected for further investigation. 
Citation: Elhassan GO, Hay YK, Woei WJ, Khan J, Alfarouq KO, et al. (2014) Preparation and In Vitro Characterization of Artemisinin FreezeDried Powders with Various Carriers. J Pharmacovigil 3: 1000156. doi:10.4172/2329-6887.1000156

Page 4 of 6

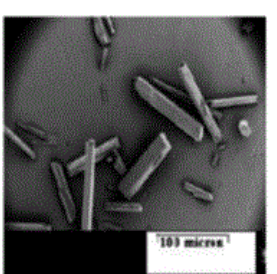

Artemisinin

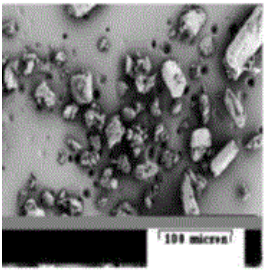

PM (ART-PVP K-25)

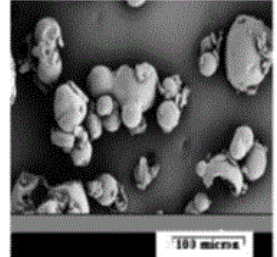

PVP K-25

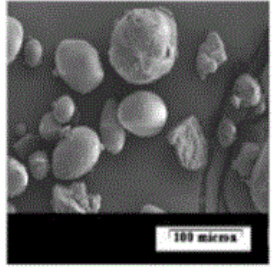

PM (ART-Dextrin)

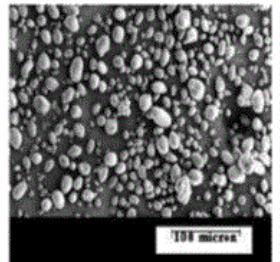

Dextrin

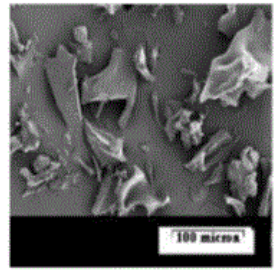

ART-PVP K-25 FD
Figure 4: Micrographs of artemisinin, carriers, physical mixture $(\mathrm{PM})$ of artemisinin-different carriers and freeze-dried (FD) product prepared of artemisinin-PVP K-25.

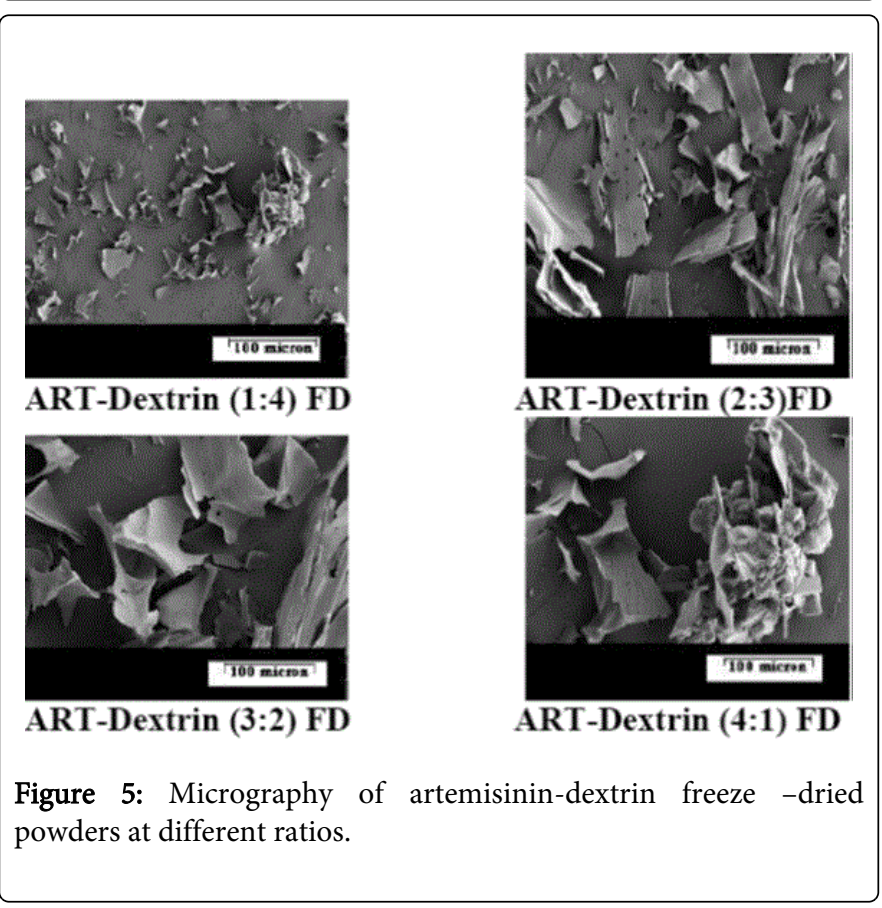

\section{Study of effect of additional cryoprotectants}

Molpeceres et al. [17] and Ozaki and Hayashi [18] reported that for freeze-dried products, addition of cryoprotectant was essential for the maintenance of the initial formulation characteristics. Therefore, in the present study, to enhance the solubility of the freeze-dried artemisinin-dextrin powder, cryoprotectants were added.

Chacön et al. [19] used mannitol and other cryoprotectants to enhance the stability of the freeze-dried products of cyclosporineloaded poly (D, L lactide glycolide). Table 1 shows the formula used for the preparation of the freeze-dried product of artemisinin-dextrin using different ratios of two cryoprotectants, mannitol or citric acid [20].

In Figure 6 it can be noticed that both citric acid and mannitol at different ratios increased the solubility of artemisinin-dextrin freezedried products. Each carrier was incorporated into the mixture of artemisinin-dextrin in water and then mixtures were freeze-dried. The freeze-dried powder containing citric acid was able to significantly increase the solubility of artemisinin compared to the one with mannitol.

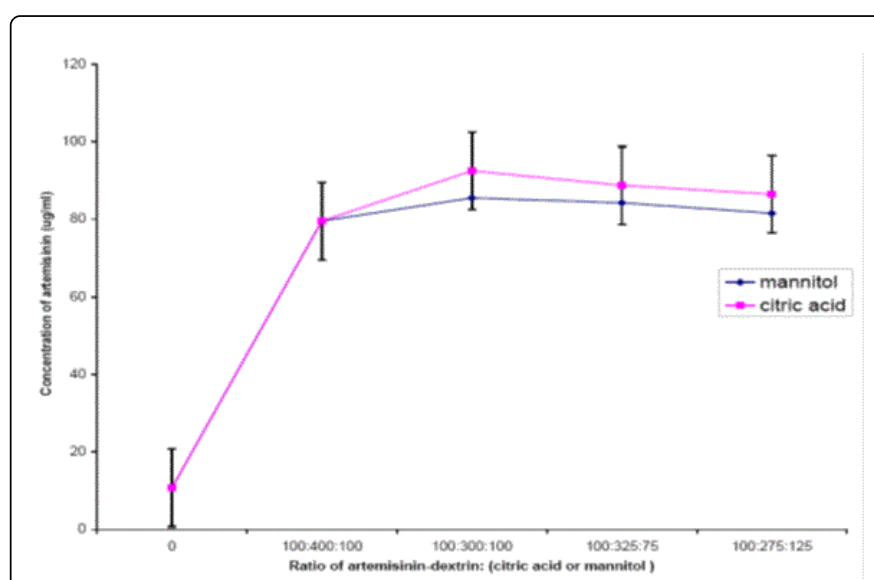

Figure 6: Artemisinin solubility in ART:dextrin:citric acid/mannitol freeze-dried powder.

The increase in solubility was well supported by SEM and DSC studies, as shown in Figures 7 and 8, respectively. In Figure 5 it can be noticed that in the artemisinin freeze-dried powders containing either citric acid (CA) or mannitol, these ingredients were present as irregular particles in which the original morphology of all components disappeared in the case of citric acid, where tiny aggregates of amorphous small pieces of irregular size were present, compared to mannitol. Therefore reduced particle size resulted in an increase in surface area responsible for enhancing the solubility of the drug [20].

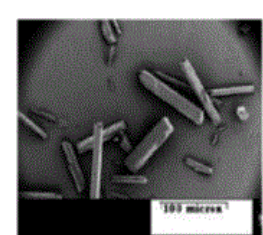

Artemisinin

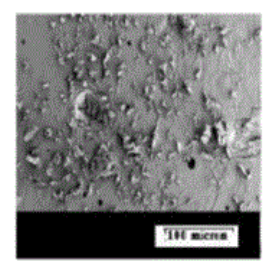

Artemisinin-Dextrin-CA (FD)

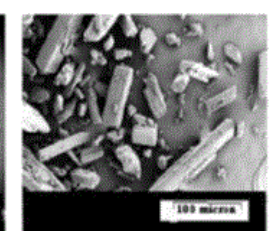

Mannitol

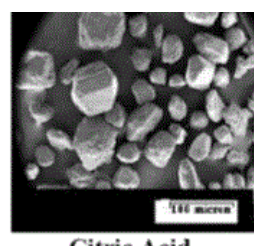

Citric Acid
Figure 7: Micrographs of pure artemisinin, mannitol, citric acid and freeze-dried product of artemisinin-dextrin-citric acid / mannitol. 


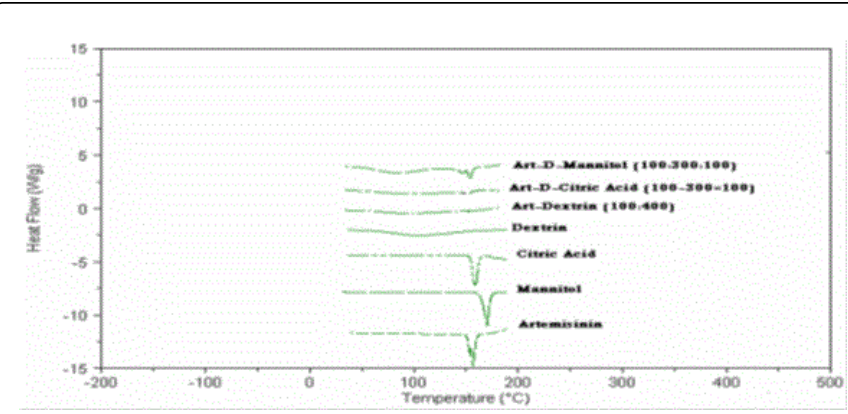

Figure 8: Thermograms of artemisinin and artemisinin-dextrin (D)-mannitol / citric acid at a ratio of 1:3:1.

Figure 5 presents the DSC curves of pure artemisinin, dextrin, citric acid, mannitol and artemisinin-dextrin freeze-dried powders containing citric acid / mannitol at a ratio of 1: 3: 1. From the thermogram it is clear that the endothermic peaks at $154.7^{\circ} \mathrm{C}, 165^{\circ} \mathrm{C}$ and $156^{\circ} \mathrm{C}$, corresponding to the melting points of pure artemisinin, mannitol and citric acid, respectively, were observed. The DSC thermogram for the freeze-dried products artemisinin-dextrin and artemisinin-dextrin-citric acid showed no characteristic endothermic peak. The disappearance of the specific peak of the drug indicates that the drug and the carrier interacted with each other [15], whereas the DSC curves of the freeze-dried product of artemisinin-dextrinmannitol recorded a peak corresponding to artemisinin melting enthalpy. This indicates that artemisinin and the carrier did not interact with each other and artemisinin was still in its crystalline form.

\section{Dissolution}

Figure 9 shows the dissolution profile of freeze-dried powders of artemisinin-dextrin-citric acid / mannitol and their physical mixtures at a ratio of 100: 300: 100 compared to pure artemisinin. From the figure it is evident that the dissolution of freeze-dried powders was faster compared to either physical mixture or drug alone. In Figure 10 it can be noticed that the freeze-dried product of artemisinin-dextrincitric acid prepared at $80^{\circ} \mathrm{C}$ displayed faster rates than freeze-dried products prepared at $60^{\circ} \mathrm{C}$ and freeze-dried products of artemisnindextrin-mannitol prepared at $60^{\circ} \mathrm{C}$ and $80^{\circ} \mathrm{C}$. The above results were in line with findings reported by Corrigan [13], showing that the physico-chemical characteristics of the drug are affected by the method of its preparation, the type and properties of the polymer used.

The high rate and the extent of dissolution from freeze-dried powder of artemisinin-dextrin may be attributed to the hydrophilic effect of dextrin, which can reduce both the hydrophobicity of artemisinin as well as interfacial tension between artemisinin and the dissolution medium, so the particle size is reduced to molecular size, and thus faster dissolution rate can be achieved.

\section{Conclusion}

From the above-mentioned results it can be concluded that the freeze-drying technique can be employed to prepare artemisnindextrin freeze-dried products capable of increasing the solubility and dissolution rate of artemisinin. Moreover, incorporation of citric acid into the mixture of artemisinin-dextrin leads to a further enhancement in the dissolution rate.

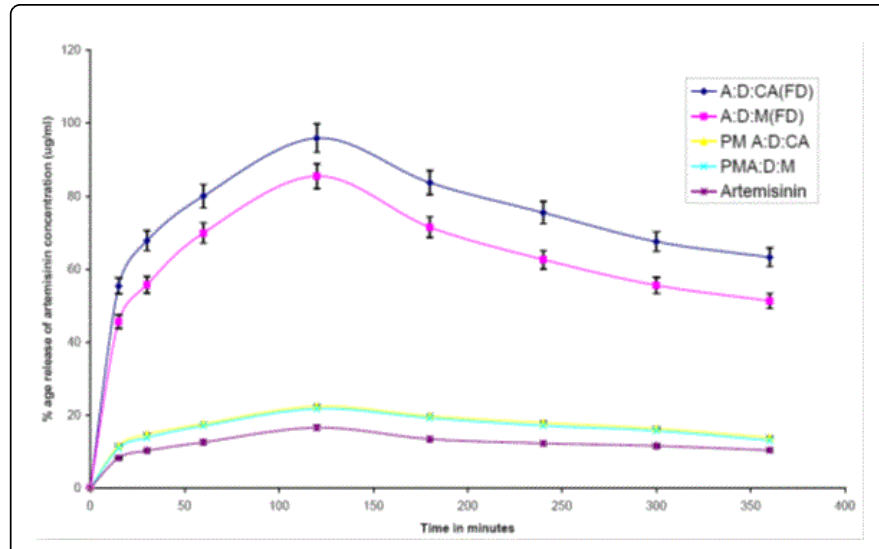

Figure 9: Compartive dissolution profile of artemisinin from freezedried product and Physical Mixture (PM) of artemisinin-dextrincitric acid / mannitol (A: D: CA/M) at ratio 1:3:1.

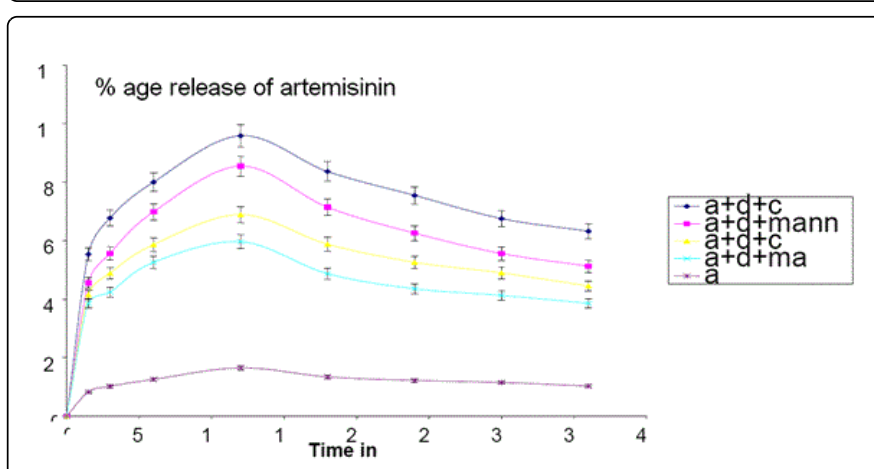

Figure 10: Comparative dissolution profile of artemisinin from pure artemisinin and in artemisinin-dextrin-citric acid / mannitol freeze-dried product (A-D-CA/M) at ratio of 1:3:1 using water at $80^{\circ} \mathrm{C}$ and $60^{\circ} \mathrm{C}$.

\section{References}

1. Klayman DL (1985) Qinghasou (Artemisinin) : An antimalaria drug from China. Science 228: 1049-1055.

2. World Health Organization (1994) The role of artemisinin and its derivatives in the current treatment of malaria. Report of an informal consultation, WHO/Mal/94.1067, Geneva.

3. Qinghasou Antimalaria Coordinating Research Group (1979) Antimarial Studies on qinghasou. Chin Med J 92: 811-816.

4. Ttulaer HAC, Zuidema J, Lugt CB (1991) Formulation and pharmacokinetics of artemisinin and its derivatives. Int J Pharm 69: 83-92.

5. Tachibana T, Nakamura A (1965) A method for preparing an aqueous colloidal dispersion of organic materials by using water-soluble polymers: Dispersion of beta-carotene by polyvinyl pyrrolidone. Kolloid-Z Polym 203: 130-133.

6. Jaccard TT, Leyder J (1985) Une Nouvelle Forme Galenique: Le Lyoc. Ann Pharm 2 : 123-131.

7. Van Eerdenburgh B, Froyen L, Martens JA, Balton N, Augustijin P, et al. (2007) Characterization and physicochemical properties and pharmaceutical performance of sucrose co-freeze-dried solid nanoparticulate powders the anti-HIV Loviride prepared by media milling. Int J Pharm 338: 198-206. 
Citation: Elhassan GO, Hay YK, Woei WJ, Khan J, Alfarouq KO, et al. (2014) Preparation and In Vitro Characterization of Artemisinin FreezeDried Powders with Various Carriers. J Pharmacovigil 3: 1000156. doi:10.4172/2329-6887.1000156

Page 6 of 6

8. Shoukri AR, Iman SA, Shamma RN (2009) In vitro and in vivo evaluation of nimesulide lyophilized orally disintegrating tablets. Eur J Pharm Biopharm 73: 162-171.

9. Iman SA, Mona HA (2007) In vitro and in vivo evaluation of fastdisintegrating lyophilized dry emulsion tablet containing griseofulvin. Eur J Pharm Sci 32: 58-68

10. Zhao SS, Zeng MY (1985) High performance liquid chromatographic determination of artemisinin (qinghaosu). Planta Medica 3: 233-237.

11. Topaloğlu Y, Yenev G, Gŏnŭllŭ U (1999) Inclusion of ketoprofen with skimmed milk by freeze drying. IL Pharmaco 54: 648-652.

12. Monkhouse DC, Lach JL (1972) Use of adsorbants in enhancing of drug dissolution II. J Pharm Sci 61 :435-1441.

13. Corrigan OI (1985) Mechanisme of fast release solid dispersion. Drug Dev Ind Pharm 11: 697-724.

14. Mooter VG, Augustigins P, Blatun N, Kinget R (1998) Physio-chemical characterization of solid dispersions of temazepam with polyethylene glycol 6000 and PVP K-30. Int J Pharm 164: 67-80.

15. Kumar SGV, Mishra DN (2005) Preparation and evaluation of solid dispersion of meloxicam with skimmed milk, Yakugaku Zasshi. The pharmaceutical society of Japan 126: 93-97.
16. Te Wierik GHP, Elssens AC, Besemer AC, Lerk CF (1993) Preparation, characterization and pharmaceutical application of linear Dextrins II. Complexation and dispersion of drug with amylodextrin by freeze-drying and kneading. Pharm Res 10: 1280-1284.

17. Molpeceres J, Guzman M, Bustamante P, Aberturas MR (1996) Exothermic-endothermic heat of solution shift of cyclosporine A related to poloxamer 188 behavior in aqueous solution. Int J Pharm 130: 75-81.

18. Ozaki K, Hayshi M (1998) The effect of glucose oligomers (maltodextrin) on freeze drying liposomes. Chem Pharm Bull 45: 165-170.

19. Chacon M, Malpeceres J, Berges L, Guzman, M, Arbeturas MR (1998) Stability and freeze drying of cyclosporine leaded poly (D, L Lactideglycolide) carriers. Eur J Pharm Sci 8: 99-107.

20. Ruan LP, Yu BY, Fu GM, Zhu D (2005) Improving the solubility of ampelopsin by solid dispersions and inclusion complexes. J Pharm Biomed Anal 38: 457-464. 